\title{
Efficacy and safety of plastic versus lumen-apposing metal stents for transmural drainage of walled-off necrosis: a retrospective single-center study
}

\author{
Surinder Singh Rana ${ }^{a}$, Ravi Sharma ${ }^{a}$, Lovneet Dhalaria ${ }^{a}$, Rajesh Guptab \\ Postgraduate Institute of Medical Education and Research (PGIMER), Chandigarh, India
}

\section{Abstract}

\section{Introduction}

Acute pancreatitis (AP) is a disease of varying severity, ranging from mild self-limiting to life-threatening

Departments of ${ }^{a}$ Gastroenterology (Surinder Singh Rana, Ravi Sharma, Lovneet Dhalaria); ${ }^{b}$ Division of Surgical Gastroenterology, Department of Surgery (Rajesh Gupta), Postgraduate Institute of Medical Education and Research (PGIMER), Chandigarh, India

\section{Conflict of Interest: None}

Correspondence to: Professor Surinder S. Rana, MD, DM, FASGE, Department of Gastroenterology, Postgraduate Institute of Medical Education and Research (PGIMER), Chandigarh 160012, India,

e-mail: drsurinderrana@yahoo.co.in

Received 8 April 2020; accepted 27 April 2020;

published online 25 May 2020

DOI: https://doi.org/10.20524/aog.2020.0499

๑ 2020 Hellenic Society of Gastroenterology severe disease associated with various systemic and local complications [1]. Walled-off necrosis (WON) is an important local complication of AP that occurs in the delayed phase of the illness. It is usually formed 4 weeks after an episode of acute necrotizing pancreatitis and is defined as an encapsulated collection of solid and liquid necrotic material $[1,2]$. Patients with WON have a variable course, with the majority of patients having an uneventful course [3]. However, a significant number of patients with WON develop symptoms that include persistent significant pain, infection, gastric outlet obstruction or biliary obstruction, and these patients require drainage. The drainage of symptomatic WON can be achieved using image-guided percutaneous, endoscopic or minimally invasive surgical means. The availability of endoscopic ultrasound (EUS) and advances in endoscopic techniques, as well as accessories, have revolutionized the field of endoscopic treatment of pancreatic fluid collections. Currently, endoscopic transmural drainage 
is considered as the mainstay of treatment for symptomatic pancreatic fluid collections $[4,5]$.

Endoscopic transmural drainage can be achieved by using either multiple plastic stents or biflanged fully covered self-expanding metal stents (BFMS). The introduction of lumen-apposing metal stents (LAMS), especially those with electrocautery-enhanced delivery system, like the Axios stent (Boston Scientific, Natick, MA, USA) and Niti-S Spaxus stent (Taewoong Medical, Gyeonggi-do, South Korea), have made the endoscopic drainage procedure simpler, with minimal technical complications. Recently, another electrocauteryenhanced BFMS (Hot Nagi Taewoong Medical, Gyenoggi-do, Korea) that has a simpler, intuitive delivery system has also been introduced [6]. The introduction of LAMS was considered as a game changer in the management of WON, because by providing a larger drainage diameter compared to plastic stents they were expected to provide effective drainage of solid necrotic material. Furthermore, as their deployment did not require large-diameter balloon dilatation of the transmural tract, they were expected to have fewer complications compared to plastic stents.

Despite this theoretical advantage, studies comparing plastic and metal stents in the management of pancreatic fluid collections have shown conflicting results [7-15]. EUS-guided drainage of WON was initially performed using plastic stents, and LAMS have only been used recently. Therefore, these retrospective comparative studies have compared 2 procedures carried out in different timeframes, with LAMS being placed in an era when centers had acquired extensive experience in endotherapy of pancreatic necrosis. In addition, the use of LAMS is associated with a higher cost and various significant adverse effects, such as pseudoaneurysm-related bleeding, stent clogging by necrotic debris, requiring multiple procedures, and stent migration $[7,8]$. Moreover, many of these studies included patients with both pseudocysts and WON, a heterogeneous study population that could induce bias in the results. In the current single-center study with a large sample size, we retrospectively compared the efficacy and safety of endoscopic transmural drainage between multiple plastic stents and LAMS.

\section{Patients and methods}

The endoscopic database of our unit was retrospectively searched for all patients who had undergone EUS-guided drainage/debridement of WON between October 2009 and March 2020. The included patients had earlier been diagnosed with acute necrotizing pancreatitis, based on the revised Atlanta classification, and had subsequently developed symptomatic WON [1]. The pancreatic WON was defined as an encapsulated collection of solid and liquid pancreatic/ peripancreatic necrosis with a well-defined wall characterized on computed tomography (CT), magnetic resonance imaging, or EUS [1]. Patients with pseudocysts as defined by revised Atlanta classification, WON with $<10 \%$ solid necrotic debris, or non-pancreatic intra-abdominal collections were excluded, as were patients who did not undergo endoscopic transmural drainage of WON. The included patients were divided into
2 groups on the basis of whether multiple plastic stents or LAMS were deployed during the initial endoscopic transmural drainage. Patient demographics, etiology of AP, size of WON, type of stent used, technical details, outcome details, and procedural complications were retrieved from the database. All the endoscopic procedures were performed after informed consent had been obtained from the patients. All the patients in the initial part of the study underwent placement of multiple plastic stents. With the availability of LAMS, patients were given a choice of LAMS or plastic stents, depending upon economic affordability and availability of health insurance. At our center, the hospital admission and treatment is subsidized but the patients have to bear the cost of stents. Therefore, we did not compare the difference in the cost of treatment between the 2 groups.

\section{EUS-guided transmural drainage}

The EUS-guided transmural drainage was performed by a single endoscopist (SSR) with the patient under conscious sedation using intravenous midazolam and pentazocine. Patients were given broad-spectrum intravenous antibiotics before the procedure and these were continued orally after the procedure until the resolution of WON. Post-procedure antibiotics were modified according to the culture and sensitivity reports of the drained fluid. EUS-guided drainage was performed using a linear scanning echoendoscope (EG3870 UTK linear echoendoscope, Pentax Inc., Tokyo, Japan, or UCT180 linear echoendoscope, Olympus Optical Co. Ltd., Tokyo, Japan). The technical details of the EUS-guided drainage procedure have been published previously by us $[16,17]$. During the initial EUS examination, the WON morphology was evaluated in detail. An attempt was made to quantify the amount of solid debris, evident as echogenic material in WON, as a percentage of the total size of the collection. This quantification was an approximate visual judgment made by the endoscopist. As mentioned above, in the initial period all patients were treated with multiple transmural plastic stents (2-5 in number, 7 or $10 \mathrm{Fr}$ diameter, and $3-7 \mathrm{~cm}$ in length). A 7-Fr nasocystic catheter (NCC) was also positioned initially alongside stents for irrigation (Fig. 1). The NCC was flushed and aspirated with $200 \mathrm{~mL}$ normal saline or hydrogen peroxide every 4-6 h. After LAMS became available, depending upon financial affordability, patients were either treated with multiple plastic stents or LAMS: NAGI stent (14 or $16 \mathrm{~mm}$ ), Taewoong Medical Co., Ltd., Seoul, Korea, or Plumber Stent (16 mm diameter), MI Tech Gyeonggi-Do, 17706, Korea, or Hot Axios stent (15 mm diameter), Boston Scientific, Natick, MA, USA (Fig. 2). Patients with peripheral WON also underwent additional percutaneous drainage of peripheral collection.

\section{Follow-up protocol after initial drainage}

An endoscopic step-up approach was followed in both groups after initial transmural drainage with either multiple 


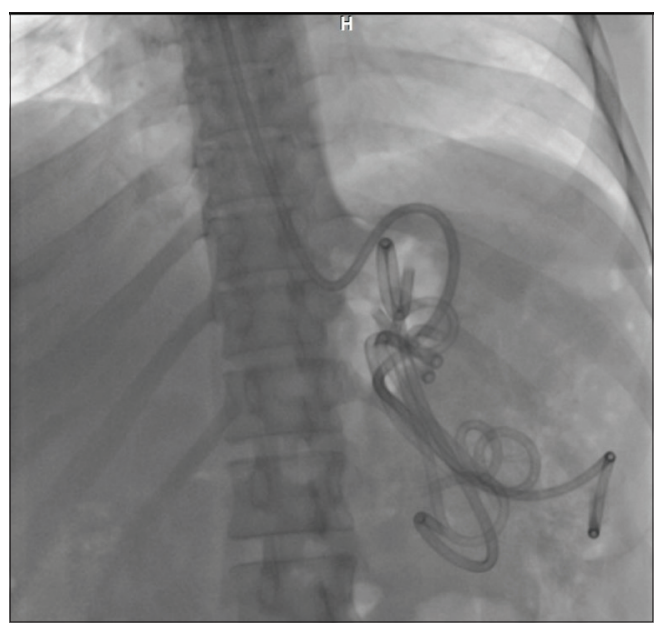

Figure 1 Endoscopic transmural drainage of walled-off necrosis with multiple plastic stents and nasocystic catheter

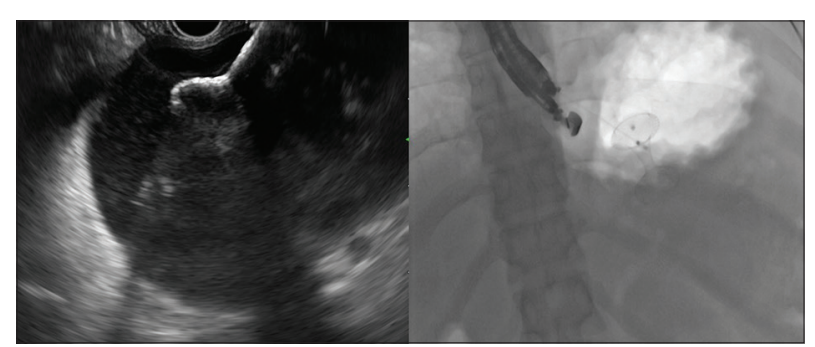

Figure 2 Endoscopic transmural drainage with lumen-apposing metal stent (Hot Axios)

plastic stents or LAMS. Patients in both groups underwent a $\mathrm{CT}$ of the abdomen $72 \mathrm{~h}$ after drainage.

\section{Multiple plastic stents group}

The NCC was removed in patients with multiple plastic stents who had symptomatic improvement with $>50 \%$ reduction in cavity size. Patients with new-onset fever or worsening of existing symptoms with persistent WON on CT underwent repeat endoscopic transmural drainage through the same tract with reinsertion of multiple plastic stents. The previously placed stents were removed, the transmural tract enlarged, and multiple plastic stents (more than on the previous occasion) were inserted. The NCC was reinserted at the discretion of the treating team. If WON persisted with persisting or worsening symptoms after a second or subsequent session of drainage, a decision concerning additional transmural drainage by multiple plastic stents, LAMS insertion, direct endoscopic necrosectomy (DEN) or surgery was taken after a review of repeat imaging findings, consultation with the patient and his/ her family, and interdisciplinary consultation with pancreatic surgeons. All non-responding patients were given a choice of LAMS insertion, and patients refusing consent for LAMS because of economic affordability underwent DEN through the dilated transmural tract. Patients who could afford LAMS underwent LAMS insertion and were subsequently managed as per the protocol of the LAMS group.

\section{LAMS group}

Patients with new-onset fever or worsening of existing symptoms with persistent WON on CT underwent repeat endoscopic transmural drainage. If the LAMS was clogged with necrotic material, the stent was de-clogged and a 7-Fr NCC was inserted through the LAMS into the necrotic cavity. The necrotic cavity was irrigated as described above. In non-responders, DEN was done through the LAMS using a combination of accessories, including grasping forceps, snare, and Dormia and Roth baskets.

\section{Treatment protocol after resolution of WON}

The follow-up imaging, including CT to document the resolution of WON, was carried out at the discretion of the treating clinician. Thefactors thathelpedin deciding thetiming of CT to document resolution of WON were clinical improvement and radiological findings on abdominal ultrasound. In patients with symptomatic improvement and resolved WON on CT, endoscopic retrograde cholangiopancreatography or magnetic resonance cholangiography was performed to delineate and define pancreatic duct disruption.

\section{Multiple plastic stents group}

In patients who had a normal pancreatic duct, all transmural plastic stents were removed, whereas in patients with partial main duct disruption, a transpapillary stent was placed across the disruption and was subsequently removed, along with the transmural stents, after resolution of the disruption had been documented. In patients who had a disconnected pancreatic duct, one or more transmural stents were left indefinitely.

\section{LAMS group}

In patients who had a normal pancreatic duct, the LAMS was removed. In patients who had partial main duct disruption, the LAMS was removed, and a transpapillary stent was placed across the disruption and was subsequently removed after resolution of the disruption had been documented. In patients who had a disconnected pancreatic duct, an attempt was made to replace the LAMS with a 7 or $10-\mathrm{Fr}$ double pigtail plastic stent.

All these patients were followed up in our outpatient clinic once every 3 months for the first year and thereafter every 6 months. Patients who failed to report to the clinic were contacted by telephone. 


\section{Outcome measures}

The various outcome measures studied included technical success, defined as the ability to place the transmural stents or LAMS into WON, confirmed radiographically and endoscopically; clinical success, defined as radiographic resolution of WON along with symptomatic improvement and occurrence of adverse events, including bleeding, perforation and unplanned endoscopic/radiological or surgical interventions.

\section{Statistical analysis}

The data were represented as percentages for categorical variables, and mean \pm standard deviation (SD) for quantitative variables. The continuous variables were compared using Student's $t$-test, whereas the categorical variables were compared using the chi-square or Fisher exact test. A p-value of $<0.05$ was considered significant.

\section{Results}

\section{Demographics and imaging characteristics}

During the study period, 166 patients (138 male [83\%]; mean age $37.5 \pm 9.1$ years) who had symptomatic WON were treated with EUS-guided transmural drainage using either multiple plastic stents or LAMS. All patients had underlying $\mathrm{AP}$ and the etiology was alcohol in 113 (68\%) patients, gallstones in $35(21 \%)$, idiopathic in $13(7.8 \%)$, and other in $5(3.2 \%)$ patients (trauma 2, post-endoscopic retrograde cholangiopancreatography 2 , and drug-induced 1 ). The patients presented to us at mean duration of $65.4 \pm 26.3$ days after the onset of episode of AP and the mean size of WON (largest diameter) on presentation was $10.8 \pm 2.6 \mathrm{~cm}$. Among the 166 patients, LAMS were used in 28 (16.8\%) patients (Plumber stent in 21, Nagi stent in 5 and Hot Axios in 2 patients respectively) and multiple plastic stents were used in $138(83.2 \%)$ patients. The median number of plastic stents inserted was 2 during the initial EUS-guided drainage and 3 during the second drainage session. The baseline demographic characteristics, the etiology of AP and the location of the WON were comparable between the 2 groups (Table 1). However, patients in whom LAMS was inserted had a significantly larger size of WON (11.9 vs. $10.6 \mathrm{~cm}, \mathrm{P}=0.009)$ and a significantly higher proportion of solid content $(40.89 \%$ vs. $30.25 \%$, $\mathrm{P}=0.00004)$. Also the timing of endoscopic intervention after an episode of AP was significantly earlier in the LAMS group than in the multiple plastic stents group (49.7 vs. 68.6 days, $\mathrm{P}=0.0002$ ) (Table 1).

\section{Outcome parameters}

\section{Resolution of WON}

The initial EUS-guided transmural drainage procedure was technically successful in all 138 patients in the multiple plastic stents group, whereas it was technically successful in $27 / 28$ (96.4\%) patients in the LAMS group. In one patient in the LAMS group, the stent became externally deployed into the stomach. The LAMS was recaptured and redeployed during the same endoscopic session. The overall rate of resolution of WON without the need for surgery was $98.1 \%$ and the mean time for resolution was 29.3 days. The WON resolution rates

Table 1 Demographic, clinical and imaging features of patients with WON

\begin{tabular}{|c|c|c|c|}
\hline Characteristics & Multiple plastic stents $(\mathrm{n}=138)$ & LAMS $(n=28)$ & P-value \\
\hline Age (years) & 37.1 & 39.2 & 0.16 \\
\hline Males & $116(84 \%)$ & $22(78.5 \%)$ & 0.57 \\
\hline $\begin{array}{l}\text { Etiology } \\
\text { Alcohol } \\
\text { Gallstones } \\
\text { Idiopathic } \\
\text { Post-ERCP } \\
\text { Drug-induced } \\
\text { Trauma }\end{array}$ & $\begin{array}{c}98(71 \%) \\
27(19.6 \%) \\
9(6.6 \%) \\
1(0.7 \%) \\
1(0.7 \%) \\
2(1.4 \%)\end{array}$ & $\begin{array}{c}15(53.5 \%) \\
8(28.6 \%) \\
4(14.3 \%) \\
1(3.6 \%) \\
0 \\
0\end{array}$ & $\begin{array}{c}0.07 \\
0.31 \\
0.23 \\
0.30 \\
>0.99 \\
>0.99\end{array}$ \\
\hline Infected WON & $53(38.4 \%)$ & $11(39.2 \%)$ & $>0.99$ \\
\hline Size of WON (cm) & 10.6 & 11.9 & 0.009 \\
\hline Duration of presentation after episode of AP (days) & 68.6 & 49.7 & 0.0002 \\
\hline $\begin{array}{l}\text { Location of WON } \\
\text { Head of pancreas } \\
\text { Body of pancreas } \\
\text { Tail of pancreas } \\
\% \text { solid debris }\end{array}$ & $\begin{array}{c}4(2.8 \%) \\
125(90.6 \%) \\
9(6.6 \%) \\
30.25 \%\end{array}$ & $\begin{array}{c}0(0 \%) \\
25(89.3 \%) \\
3(10.7 \%) \\
40.89 \%\end{array}$ & $\begin{array}{c}>0.99 \\
0.73 \\
0.42 \\
0.00004\end{array}$ \\
\hline
\end{tabular}

WON, walled-off necrosis; LAMS, lumen-apposing metal stents; ERCP, endoscopic retrograde cholangiopancreatography; AP, acute pancreatitis 
did not differ between the multiple plastic stents and LAMS groups $(98.5 \%$ vs. $96.4 \%$ respectively, $\mathrm{P}=0.42)$. However, the time taken to resolution was significantly shorter in the LAMS group than in the multiple plastic stents group (26.7 vs. 29.8 days, $\mathrm{P}=0.03)$. The need for $\mathrm{DEN}$ was comparable between the 2 groups (25\% in LAMS vs. $11.5 \%$ in multiple plastic stents group, $\mathrm{P}=0.07)$. Although statistically non-significant, the greater need for DEN in the LAMS group could be because of the significantly larger size of WON and significantly higher proportion of solid content in the LAMS group. The mean number of endoscopic procedures required for the resolution of WON was comparable between the 2 groups ( 3.33 in LAMS vs. 3.53 in multiple plastic stents group, $\mathrm{P}=0.28$ ). Following resolution of WON, a transmural double pigtail plastic stent was left in situ in all patients with a disconnected pancreatic duct in the multiple plastic stents group, whereas this stent was placed successfully in only $12 / 20(60 \%)$ such patients in the LAMS group. In the remaining $8(40 \%)$ patients, the LAMS could not be replaced with plastic stents because of complete collapse of the WON cavity.

\section{Complications}

There was no significant difference in the mortality rates between the 2 groups $(0.7 \%$ for multiple plastic stents vs. $3.5 \%$ in LAMS group, $\mathrm{P}=0.30)$. The need for rescue surgery was also comparable between the 2 groups $(0.7 \%$ for multiple plastic stents vs. $0 \%$ in LAMS group, $\mathrm{P}>0.99)$. The rates of other complications, including pneumoperitoneum (1.5\% for multiple plastic stents vs. $\%$ in LAMS group, $\mathrm{P}>0.99$ ) and bleeding requiring surgery or radiological intervention (2.8\% for multiple plastic stents vs. $7 \%$ in LAMS group, $\mathrm{P}=0.26$ ), were also comparable between the 2 groups. Selflimiting pneumoperitoneum occurred in 2 patients in the multiple plastic stents group and was successfully managed conservatively with nasojejunal tube feeding and antibiotics.

\section{Discussion}

With the advent of EUS and the availability of improved accessories, endoscopic transmural drainage has become the preferred modality of treatment of both pancreatic pseudocysts and WON. Despite a number of studies showing its superiority over percutaneous as well as minimally invasive surgical drainage, the choice of stents for transmural drainage continues to be a topic of debate. LAMS were presumed to be better than multiple plastic stents, as they provide a larger drainage diameter expected to provide effective drainage of solid necrotic material. However, various studies and metaanalyses that have compared plastic and metal stents for the treatment of WON have shown conflicting results in terms of efficacy and adverse events [6-14].

Abu Dayyeh et al retrospectively compared 36 patients who underwent endoscopic transmural drainage with multiple plastic stents and 58 patients who underwent metal stent placement [18]. They reported that the WON resolution rates were comparable between the 2 groups ( $91.7 \%$ for plastic stents vs. $94.8 \%$ for metal stents, $\mathrm{P}=0.55)$. However, spontaneous resolution of WON without need of DEN was significantly more common in the metal stent group $(30.8 \%$ vs. $60.4 \%, \mathrm{P}=0.01)$. They also reported that the complications were similar in both groups except for clinically significant bleeding requiring endoscopic intervention, that was more common in the plastic stent group ( $14 \%$ vs. $2 \%, \mathrm{P}=0.02)$. Wang et al retrospectively evaluated the efficacy of various types of stents in 160 patients with pancreatic fluid collections (62 patients drained with plastic stents, 28 patients with fully covered self expanding metal stents, and 70 patients with LAMS) [13]. They also reported that the technical success $(93.5 \%$ vs. $96.4 \%$ vs. $94.3 \%, \mathrm{P}>0.99)$ and treatment success rates $(84.6 \%$ vs. $85.2 \%$ vs. $89.2 \%, \mathrm{P}=0.763)$ were similar among the 3 groups. However, clinically significant bleeding occurred only in the metal stents group.

Siddiqui et al also retrospectively compared 106 patients with WON drained using plastic stents with 121 patients drained using fully covered self-expanding metallic stents and 86 patients drained using LAMS [15]. The technical success rates were similar among the 3 groups, but the clinical resolution was lower in the plastic stent group compared to the self-expanding metallic stent and LAMS groups (81\% vs. $95 \%$ vs. $90 \%, \mathrm{P}=0.001)$. The mean number of procedures required for WON resolution was also found to be significantly lower in the LAMS group than in the other 2 groups (2.2 vs. 3 vs. 3.6 respectively, $\mathrm{P}=0.04)$. Ge et al retrospectively compared 78 patients who underwent transmural drainage of WON using plastic stents with 34 patients who underwent transmural drainage using LAMS [19]. They also reported that WON resolution was similar between the LAMS and plastic stents groups $(94.1 \%$ vs. $92.1 \%, \mathrm{P}=0.510)$, as was the number of procedures ( 1.5 vs. $1.5, \mathrm{P}=0.871)$. However, LAMS were associated with faster resolution ( 86.9 vs. 133.6 days, $\mathrm{P}=0.038$ ), and a lower requirement for surgery ( $0 \%$ vs. $12.8 \%, \mathrm{P}=0.031)$.

Bang et al reported a randomized study comparing 31 patients with WON treated with LAMS with 29 patients who underwent placement of multiple plastic stents [7]. They reported that there was no significant difference between the 2 groups in the total number of procedures performed (median 2 [range 2-7] for LAMS vs. 3 [range 2-7] for plastic, $P=0.192$ ), treatment success, clinical adverse events, readmissions or overall treatment costs. Similar to the abovementioned studies, the systematic reviews and meta-analyses have also yielded varying results. Mohan et al reported that the pooled rate of clinical-success as well as adverse events was similar between LAMS and plastic stents, with pooled success rates being $88.5 \%$ in the LAMS group and $88.1 \%$ in the plastic stent group [9]. On the other hand, Saunders et al reported that metal stents for the drainage of pancreatic fluid collections were associated with better clinical success, fewer complications and less bleeding compared to plastic stents [20]. Yoon et al, in their meta-analysis, also reported that metal stents had a higher clinical success rate (odds ratio [OR] 3.39, 95\% confidence interval [CI] 2.05-5.60) and a lower overall adverse event rate (OR 0.37, 95\%CI 0.21-0.66) than plastic stents [21]. Moreover, 
in subgroup analyses, the authors reported that metal stents demonstrated superiority over plastic stents for treatment of both pseudocyst (OR 5.35, 95\%CI 1.35-21.19) and WON (OR 3.37, 95\%CI 1.89-5.99).

In our study, we also found that the technical success and resolution rates of WON were comparable in both groups. Unlike previous studies, we also evaluated the morphology of WON in detail, including the quantification of solid necrotic material in WON in both groups, as the amount of necrotic material has important therapeutic implications [22]. Although there was significantly greater necrotic content, as well as a larger size of WON, in the LAMS group compared to the plastic stents group, the resolution rates, number of endoscopic procedures required for resolution, complications and mortality were comparable in both groups. The time taken to resolution was significantly shorter in the LAMS group than in the plastic stent group. Although the need for DEN was non-significantly greater in the LAMS group compared to the plastic stent group ( $25 \%$ vs. $11.5 \%, \mathrm{P}=0.07$ ), this difference could have been due to the significantly larger size of WON and significantly higher proportion of solid content in the LAMS group. Parsa et al compared a larger diameter $(20 \mathrm{~mm})$ LAMS with a standard $15 \mathrm{~mm}$ LAMS in 306 patients with symptomatic WON and reported that patients who received the $20 \mathrm{~mm}$ LAMS required fewer DEN sessions (mean 1.3 vs. 2.1, $\mathrm{P}<0.001)$ [23].

We also found that procedural complications, including pneumoperitoneum and bleeding requiring surgery or radiological intervention, were comparable between the 2 groups. An international multicenter comprehensive analysis of adverse events associated with LAMS placement for pancreatic fluid collection drainage reported that mild, moderate and severe complications occurred in $25.3 \%, 68.4 \%$ and $6.3 \%$ of patients, respectively [24]. These results suggest that LAMS are not a panacea for the management of WON and can be associated with significant adverse effects. We also found that in $40 \%$ of patients with a disconnected pancreatic duct in the LAMS group the metal stents could not be replaced with a long-term transmural plastic stent because of complete collapse of the WON cavity; the long-term consequences of this need to be studied.

Our study had several limitations. It was a single-center retrospective study in which more patients were treated with multiple plastic stents than with LAMS. Although the baseline demographics and etiology of AP were comparable between the 2 groups, patients in the LAMS group had significantly larger sized WON with more solid necrotic content. Despite these adverse baseline WON features, the resolution rates, complications and mortality were comparable in both groups. Another limitation is that the number and size of plastic stents inserted in patients were variable. Moreover, at our center the hospital admission and treatment are subsidized and it is not possible to determine the actual cost of treatment; therefore, we did not compare the difference in the cost of treatment between the 2 groups.

In conclusion, the technical success, resolution rates of WON and adverse events were similar in patients treated with multiple plastic stents and LAMS. However the use of LAMS was associated with a significantly shorter time to resolution.

\section{Summary Box}

\section{What is already known:}

- Endoscopic management of walled-off necrosis (WON) involves the use of either multiple plastic stents or lumen-apposing metal stents (LAMS)

- Various studies and meta-analyses that have compared plastic and metal stents for the treatment of WON have shown conflicting results in terms of efficacy and safety

\section{What the new findings are:}

- The resolution rates of WON were similar in patients treated with multiple plastic stents and LAMS

- However, LAMS was associated with a significantly shorter time to resolution

- The complication rates, including clinically significant bleeding, were similar between multiple plastic stents and LAMS

- In $40 \%$ of patients with a disconnected pancreatic duct in the LAMS group the metal stents could not be replaced with a long-term transmural plastic stent because of complete collapse of the WON cavity

\section{References}

1. Banks PA, Bollen TL, Dervenis C, et al; Acute Pancreatitis Classification Working Group. Classification of acute pancreatitis-2012: revision of the Atlanta classification and definitions by international consensus. Gut 2013;62:102-111.

2. Rana SS. An overview of walled-off pancreatic necrosis for clinicians. Expert Rev Gastroenterol Hepatol 2019;13:331-343.

3. Rana SS, Sharma RK, Gupta P, Gupta R. Natural course of asymptomatic walled off pancreatic necrosis. Dig Liver Dis 2019;51:730-734.

4. Bang JY, Arnoletti JP, Holt BA, et al. An endoscopic transluminal approach, compared with minimally invasive surgery, reduces complications and costs for patients with necrotizing pancreatitis. Gastroenterology 2019;156:1027-1040.

5. Haney CM, Kowalewski KF, Schmidt MW, et al. Endoscopic versus surgical treatment for infected necrotizing pancreatitis: a systematic review and meta-analysis of randomized controlled trials. Surg Endosc 2020 Feb 28 [Epub ahead of print]. doi: 10.1007/ s00464-020-07469-9

6. Lakhtakia S, Nabi Z, Moon JH, et al. Endoscopic drainage of pancreatic fluid collections by use of a novel biflanged stent with electrocautery-enhanced delivery system. VideoGIE 2018;3:284-288.

7. Bang JY, Navaneethan U, Hasan MK, Sutton B, Hawes R, 
Varadarajulu S. Non-superiority of lumen-apposing metal stents over plastic stents for drainage of walled-off necrosis in a randomised trial. Gut 2019;68:1200-1209.

8. Brimhall B, Han S, Tatman PD, et al. Increased incidence of pseudoaneurysm bleeding with lumen-apposing metal stents compared to double-pigtail plastic stents in patients with peripancreatic fluid collections. Clin Gastroenterol Hepatol 2018;16:1521-1528.

9. Mohan BP, Jayaraj M, Asokkumar R, et al. Lumen apposing metal stents in drainage of pancreatic walled-off necrosis, are they any better than plastic stents? A systematic review and meta-analysis of studies published since the revised Atlanta classification of pancreatic fluid collections. Endosc Ultrasound 2019;8:82-90.

10. Hammad T, Khan MA, Alastal Y, et al. Efficacy and safety of lumen-apposing metal stents in management of pancreatic fluid collections: are they better than plastic stents? A systematic review and meta-analysis. Dig Dis Sci 2018;63:289-301.

11. Mukai S, Itoi T, Baron TH, et al. Endoscopic ultrasound-guided placement of plastic vs. biflanged metal stents for therapy of walled-off necrosis: a retrospective single-center series. Endoscopy 2015;47:47-55.

12. Lang GD, Fritz $C$, Bhat $T$, et al. EUS-guided drainage of peripancreatic fluid collections with lumen-apposing metal stents and plastic double-pigtail stents: comparison of efficacy and adverse event rates. Gastrointest Endosc 2018;87:150-157.

13. Wang Z, Zhao S, Meng Q, et al. Comparison of three different stents for endoscopic ultrasound-guided drainage of pancreatic fluid collection: A large retrospective study. J Gastroenterol Hepatol 2019;34:791-798.

14. Chen YI, Yang J, Friedland S, et al. Lumen apposing metal stents are superior to plastic stents in pancreatic walled-off necrosis: a large international multicenter study. Endosc Int Open 2019;7:E347-E354.

15. Siddiqui AA, Kowalski TE, Loren DE, et al. Fully covered selfexpanding metal stents versus lumen-apposing fully covered selfexpanding metal stent versus plastic stents for endoscopic drainage of pancreatic walled-off necrosis: clinical outcomes and success. Gastrointest Endosc 2017;85:758-765.

16. Rana SS, Bhasin DK, Rao C, Sharma R, Gupta R. Consequences of long term indwelling transmural stents in patients with walled off pancreatic necrosis and disconnected pancreatic duct syndrome. Pancreatology 2013;13:486-490.

17. Rana SS, Sharma V, Sharma R, Gupta R, Bhasin DK. Endoscopic ultrasound guided transmural drainage of walled off pancreatic necrosis using a "step - up" approach: A single centre experience. Pancreatology 2017;17:203-208.

18. Abu Dayyeh BK, Mukewar S, Majumder S, et al. Large-caliber metal stents versus plastic stents for the management of pancreatic walled-off necrosis. Gastrointest Endosc 2018;87:141-149.

19. Ge PS, Young JY, Jirapinyo P, Dong W, Ryou M, Thompson CC. Comparative study evaluating lumen apposing metal stents versus double pigtail plastic stents for treatment of walled-off necrosis. Pancreas 2020;49:236-241.

20. Saunders R, Ramesh J, Cicconi S, et al. A systematic review and meta-analysis of metal versus plastic stents for drainage of pancreatic fluid collections: metal stents are advantageous. Surg Endosc 2019;33:1412-1425.

21. Yoon SB, Lee IS, Choi M-G. Metal versus plastic stents for drainage of pancreatic fluid collection: a meta-analysis. United European Gastroenterol J 2018;6:729-738.

22. Rana SS, Bhasin DK, Sharma RK, et al. Do the morphological features of walled off pancreatic necrosis on endoscopic ultrasound determine the outcome of endoscopic transmural drainage? Endosc Ultrasound 2014;3:118-122.

23. Parsa N, Nieto JM, Powers P, et al. Endoscopic ultrasound-guided drainage of pancreatic walled-off necrosis using 20 -mm versus 15 $\mathrm{mm}$ lumen-apposing metal stents: an international, multicenter, case-matched study. Endoscopy 2020;52:211-219.

24. Fugazza A, Sethi A, Trindade AJ, et al. International multicenter comprehensive analysis of adverse events associated with lumenapposing metal stent placement for pancreatic fluid collection drainage. Gastrointest Endosc 2020;91:574-583. 